\title{
Allées critiques au Jardin des Plantes
}

Joëlle Gleize

\section{(2) OpenEdition}

Journals

Édition électronique

URL : http://journals.openedition.org/ccs/1517

DOI : $10.4000 /$ ccs. 1517

ISSN : 2558-782X

\section{Éditeur :}

Presses universitaires de Rennes, Association des lecteurs de Claude Simon

\section{Édition imprimée}

Date de publication : 30 août 2018

Pagination : 65-93

ISBN : 978-2-7535-7489-2

ISSN : 1774-9425

Référence électronique

Joëlle Gleize, «Allées critiques au Jardin des Plantes », Cahiers Claude Simon [En ligne], 13 | 2018, mis en ligne le 30 août 2019, consulté le 20 février 2020. URL : http://journals.openedition.org/ccs/1517 ; DOI : $10.4000 /$ ccs. 1517 


\title{
ALLÉES CRITIQUES AU JARDIN DES PLANTES
}

\author{
Joëlle GLEIZE \\ Université Aix-Marseille
}

Au moment de la sortie du Jardin des Plantes, en 1997, seize ans après la publication des Géorgiques, la critique simonienne est entrée dans sa phase de maturité, comme l'atteste le volume collectif dirigé par Ralph Sarkonak, La Réception critique ${ }^{1}$. Les années 80 ont vu progressivement disparaître le principal tabou qui pesait sur elle, celui du référent - en particulier celui du matériau autobiographique -, et cela en raison de l'évolution de l'œuvre elle-même: depuis Les Géorgiques (1981), qui renouent avec la matière biographique familiale et autographique des premiers livres, les romans de Simon (et L'Invitation qui ne se dit pas tel) désignent, voire affichent leurs archives et leur référence au monde, à l'histoire personnelle, familiale et collective. Depuis que l'auteur luimême a souligné la part de "vécu " à la base de son écriture ${ }^{2}$, les questionnements se sont ouverts aux relations de l'œuvre à son auteur. Ouverture qui se conjugue avec la diversification des questionnements critiques, plus soucieux de rigueur que de formalisme théorique. La singularité de ce "roman ", puisque tel est le sous-titre du Jardin des Plantes, fait franchir un seuil à la critique simonienne. "C'est la première fois que Simon évoque autant d'aspects différents de sa vie " souligne Jean H. Duffy (2006: 1466). Tout en étant très différent de L'Acacia paru huit ans plus tôt, il relève bien de ce dernier pan de l'œuvre où disparaitt toute trace de fiction, en même temps que ses spécificités formelles, fragmentation et polyphonie visibles, sont si ostensibles qu'elles attirent l'atten-

1. Revue des Lettres modernes, série "Claude Simon", n 6 ("La Réception critique »), Caen, Minard, 2012.

2. Simon affirme que tous ses livres sont "à base de vécu " dans son entretien avec Marianne Alphant, en 1989, à propos de L'Acacia, "À quoi bon inventer? ", Libération, 31 août 1989 (repris dans les Cahiers Claude Simon, $\mathrm{n}^{\circ} 11,2016$, p. 19-24). 
tion critique, ce qui va favoriser aussi bien l'étude des références historiques ou intertextuelles que celle de la forme discontinue.

Les conditions de lecture du texte - place dans l'œuvre, images de l'auteur et autonomisation du livre - déterminent fortement les questions posées. Depuis la publication des Géorgiques et de L'Acacia, la critique française s'est autorisée ce que la critique anglo-saxonne pratiquait déjà ${ }^{3}$ : la prise en compte des matériaux autobiographiques et historiques. Sans doute la question du référent et du document suscitait-elle encore des tensions et parfois de la part de l'auteur lui-même, comme le montre Jean-Yves Laurichesse (2012). Mais elle a pris une place majeure et contribué à rendre plus riche et complexe le discours critique. Claude Simon a largement encouragé cette évolution en publiant de nombreux documents autobiographiques et photographiques dans l'ouvrage à tirage limité Album d'un amateur en 1988, puis dans la revue suisse $D u^{4}$ en 1999 . L'évolution de son écriture, qui n'en continue pas moins à éviter les pièges de la représentation, avait été déterminante dans l'intérêt critique porté au rapport de l'œuvre au monde. Le Jardin des Plantes va dans le même sens: il accorde une place éminente à l'entretien de l'auteur $S$. avec un journaliste et aux questions du référent et des rapports œuvre-vie, promues lignes de force d'un livre où abondent citations de textes et documents divers.

Peut-on, dans ces vingt ans de lectures du Jardin des Plantes, déceler des seuils susceptibles d'infléchir la critique - sans que les effets en soient pour autant explicitables? La parution du Tramway, en 2001, modifie ou confirme le regard sur la singularité de ce "roman »: il ne couronne plus, sans la sommer ni la clore, l'œuvre simonienne, mais opère un écart par rapport à ce qu'on a appelé " roman généalogique » ou roman familial, en revenant à une recherche plus formelle 5 . Cependant c'est 2005 qui constitue à l'évidence un seuil majeur. Avec la disparition de l'auteur, l'œuvre (du moins sa part éditée) se clôt et chaque roman se fige à sa place; c'est aussi une présence forte, intimidante et virtuellement critique, qui disparaît - une présence dont l'impact sur les études publiées échappe par ailleurs à toute saisie ${ }^{6}$. Les livres, dès lors, et Le Jardin des

3. En témoignent l'ouvrage collectif dirigé par Alastair B. Duncan, Claude Simon, New Directions (Edinburgh, Scottish Academic Press, 1985), et, cinq ans plus tard, la livraison de la Revue des Sciences Humaines coordonnée par Guy Neumann ( $n^{\circ} 220$, oct.-déc. 1990) et consacrée à Simon.

4. Album d'un amateur, Remagen-Rolandseck, Rommerskirchen, 1988 et Du, die Zeitschrift der Kultur, Zurich, n 691, janv. 1999.

5. Ce que confirme son insertion voulue par Simon dans le premier volume de la Pléiade, d'où sont écartés les romans les plus fortement "à base de vécu ".

6. La réprobation manifestée par Claude Simon devant telle ou telle interprétation pouvait-elle contrecarrer la réhabilitation du référent qu'il avait cependant lui-même autorisée? La question reste ouverte. 
Plantes avec eux, s'autonomisent et deviennent plus encore objets d'appropriation possible pour les lecteurs.

L'image de l'auteur Claude Simon s'est aussi modifiée notablement depuis 1997: le prix Nobel y a certes contribué, mais aussi la publication de son œuvre, en 2006 et 2013, dans la Bibliothèque de la Pléiade. Ainsi doublement consacrée, l'œuvre est devenue un terrain de recherche très prisé; les travaux, articles et thèses, se multiplient - dont les auteurs n'ont jamais côtoyé Claude Simon: un phénomène désormais plus français qu'anglo-saxon, la recherche anglo-saxonne, longtemps pionnière, privilégiant d'autres objets: littérature francophone et extrême contemporain (voir Laurichesse 2012 et Dirkx et Mougin 2011). Le nom de Simon est désormais celui d'un "auteur majeur" du siècle précédent, et, malgré - ou avec - toute sa puissance d'innovation et de transformation de la littérature en train de s'écrire, celui d'un "classique " au sens de consacré par les institutions (scolaire, éditoriale, culturelle ${ }^{7}$ ). Ce nom désigne dès lors pour beaucoup une œuvre plus qu'un homme, un univers textuel multiple et d'une richesse inépuisable.

En témoigne l'abondante fortune critique du Jardin des Plantes, faite essentiellement d'articles, mais aussi de trois volumes collectifs et parfois d'un chapitre d'ouvrage, mais pas encore d'un ouvrage entier. Nous présenterons d'abord les approches internes, qu'elles soient formelles ou thématiques, puis, les travaux sur toutes les formes d'intertextualité, depuis la réécriture et la réflexivité jusqu'aux études intersémiotiques, au sens restreint d'interaction entre la littérature et le cinéma, enfin les études des relations du texte avec ses référents, l'histoire familiale ou collective et le contexte géographique ou culturel, dernier chantier à s'être ouvert sur l'œuvre.

\section{LECTURES INTERNES}

Même si, comme le soulignent Paul Dirkx et Pascal Mougin, la critique simonienne s'est largement ouverte depuis les années quatre-vingt-dix à des questionnements plus diversifiés, "la perspective demeure largement intratextuelle ( Dirkx et Mougin 2011: 13) tout en témoignant de ce formalisme critique apaisé et ouvert que constatait Hannes de Vriese dans le bilan qu'il a effectué des études sur L'Acacia (Vriese 2016: 34).

7. Voir A. Viala, "Qu'est-ce qu'un classique?", Bulletin des Bibliothèques de France, t. 37, n 1, 1992. L'inscription au programme de l'agrégation de lettres puis de l'entrée à l'École normale supérieure de La Route des Flandres, en 1998, en exposant le texte aux exercices académiques et en le consacrant comme œuvre patrimoniale, a fortement contribué à détacher le livre de son auteur. 


\section{Lectures des formes}

Les études de poétique ou de stylistique consacrées au Jardin des Plantes comptent, avec les critiques thématiques, parmi les premières. Et cela n'étonnera pas, tant l'innovation formelle saute aux yeux, dans ce livre dont la mise en page bouleverse tous les codes, où une fragmentation irrégulière mais constante dans les trente-trois premières pages dispose et disperse les blocs encadrés de blanc. Questionnement des formes, donc, pris dans la tension familière à tout lecteur de Simon entre ordre et désordre, mais sans formalisme strict, tant ces formes engagent à l'évidence une esthétique, une éthique, une vision du monde et de soi. La critique s'attache tout autant à comprendre l'éclatement des fragments qu'à éclairer ce qui les tient ensemble et leur permet de faire livre: les liaisons, les échos, les ruptures, les reprises, les contrepoints, etc., tout ce qui compose un ordre.

Quelques études portent sur des formes caractéristiques de l'écriture de Simon souvent étudiées, telles la description ou les métaphores; on note peu d'études du style au sens strict, ce qui peut tenir à l'extrême diversité d'écriture des fragments, le livre, à l'exemple du Jardin parisien et de ses collections végétales, accordant une importance inusitée à des citations allographes. Pour Matei Chihaia, qui étudie l'enjeu esthétique et éthique de la métaphore simonienne multipliant «les figures d'incertitude et de superpositions " (2006:97), le choix du corpus semble secondaire. C'est en revanche une mise en œuvre spécifique de la description qu'analyse Michel Bernard, dans "L'hypotypose de la place Monge » (1999) : il met en relation la description "pseudo-réaliste » d'un lieu parisien anonyme avec la réflexion ironique sur le débat de Cerisy-la-Salle où Simon avait été accusé d'exhiber ses référents. Tout en critiquant le déni de la référentialité, le critique remarque combien il est illusoire de croire dire le réel sans passer par sa représentation, donc sa transposition. Mireille Calle-Gruber (2001) s'intéresse moins à la référence qu'à la dimension réflexive du roman, et à une description qui est à la fois interprétation du réel, prise de position sur la description et critique du jugement de Breton, et qui entraîne l'imaginaire, en un "inventaire du monde sensible » (ibid.: 16), qui, grâce à la fonction de cadrage des blancs typographiques, "fait de chaque description une œuvre d'art " (ibid.: 21). En revanche, c'est au roman d'un voyageur à travers le monde que s'attache Jean-Yves Laurichesse, lisant dans les descriptions de paysages l'effet d'intensification des perceptions produit par un point de vue mobile - voiture ou avion. Il note le regard à la fois « distant et fasciné » (Laurichesse 2015: 55) dont rend compte le jeu de la métaphore qui abolit le réel ou opère un retour à l’origine dans la vision aérienne. 
S'agissant d'un livre où Yves Peyré voit « l'aveu au grand jour d'une méthode de composition la plupart du temps à peine susurrée, là fortement revendiquée " (Peyré 2004: 93-4), l'étude stylistique de l'assemblage simonien comme d'un espace où " le global et le local ne cessent d'interagir " (Yocaris et Zemmour 2015 : 199) s'avère particulièrement pertinente. Dès lors, peut-on encore parler de la composition du roman comme d' " un bloc indivisible " au sens d'une composition " fermée sur elle-même ${ }^{8}$ ", formule jusqu'alors rectrice? La tension est ici maximale entre éclatement et assemblage, mais le mode de composition reste inchangé. Dans leur étude stylistique de l'écriture simonienne, Ilias Yocaris et David Zemmour distinguent quatre procédés de composition: des composantes structurales " expressives » qui ont un sens métaphorique ou indiciel et renvoient à un monde fragmenté; le recours systématique à certaines figures de style (métaphore, épanorthose etc.); la mise en place de réseaux analogiques reliant à distance différentes composantes textuelles (données autographes et discours allographes compris); le recours à des mises en abyme. Tels sont bien les procédés qu'évoquent fréquemment les études formelles du Jardin des Plantes, à des degrés divers.

Fragmentation et composition retiennent l'attention critique dès la première publication collective consacrée au Jardin des Plantes et suscitent des lectures diverses et pourtant complémentaires. Ainsi Isabelle Serça (1999) éclaire la composition en damier du livre par la description des tableaux de Novelli autant que par l'exergue de Montaigne. Elle note le caractère hétérogène des " cases » dont varient les formes, la longueur et le genre et que délimitent des blancs; abordant le roman comme montage, elle analyse les déplacements, les coupures avec reprise, les glissements et surimpressions qui déjouent la linéarité et spatialisent la lecture. S'intéressant elle aussi au montage, Catherine Rannoux explore de façon très approfondie « le paradoxe de cette écriture de la mémoire: hantée par le temps qui la constitue, elle cherche à restituer la complexité de celui-ci, tout en feignant d'échapper à la dimension temporelle par le recours au jeu dans l'espace» (Rannoux 2000: 250). La critique note le diptyque formé par la disposition typographique du début et le scénario de la fin, où elle voit deux tentatives différentes pour écrire la simultanéité d'images mémorielles et de visions disparates. Elle souligne enfin le parti pris esthétique et la conception du sujet sous-jacents à cette mosaïque énonciative qui en appelle à la collaboration du lecteur et à ses mémoires, du texte, de l'œuvre. Dans un article dont il sera question plus loin, Sjef Houppermans observe les trois figures du montage

8. C. Simon, «Un bloc indivisible ", réponse à la question " Pourquoi des romans? ", Les Lettres françaises, $\mathrm{n}^{\circ} 740,4-10$ déc. 1958 , p. 5. 
(Houppermans 2001 : 137) que présente le roman: d'abord un échafaudage de fragments suivi d'un récit aplani, puis des paragraphes séparés de blancs dans les deux chapitres centraux, enfin le scénario, lu comme une mise en abyme ou variante supplémentaire. À partir des expériences de la peur du protagoniste et des descriptions des saisons, Ralph Sarkonak (2000) construit une lecture allégorique de la composition qu'il voit structurée par le chiffre 4; les quatre saisons: celles de l'année, celles d'une vie, d'une ouvre et du roman lui-même. Il note les parallélismes et contrastes simultanéistes du printemps, mais aussi des autres saisons, aux éléments plus dispersés mais tout aussi contrastés. Mireille Calle-Gruber prête aussi à ces contrastes une fonction structurante du livre, parlant d'un "montage en contrepoints" (Calle-Gruber 2000: 49). À partir de ces tensions internes, Jan Baetens élargit le propos en suivant les stratégies mises en œuvre depuis L'Herbe pour tenter de contourner la linéarité de l'écrit grâce aux "mécanismes contraires de la brisure et de la jonction " (Baetens 2001: 31); il voit une synthèse et une métamorphose de ces techniques dans Le Jardin des Plantes qui innove par la crudité des juxtapositions de sa mise en page, elle-même traversée de tensions entre principes de dislocation et de cohésion linéaire. Un élargissement de l'étude de la fragmentation aux deux derniers romans de Simon est aussi mené par Katerine Gosselin qui remarque une évolution entre Le Jardin des Plantes où «le narrateur fait le portrait du sujet comme somme fragmentée et mobile de ruines, laissant le lecteur imaginer des reconstitutions possibles " (Gosselin 2009: 172), et Le Tramway où disparaîtraient cette mobilité et cette ouverture.

\section{Motifs et réseaux analogiques}

Parmi les premières publications sur Le Jardin des Plantes avec les lectures des formes, les lectures thématiques suivent les réseaux que tel ou tel motif permet de construire, ces réseaux analogiques qui relient à distance les composantes dont parlaient I. Yocaris et D. Zemmour (2015). Tels les motifs de l'œil et de la queue ocellée du paon, qu’Annie Clément-Perrier (1998) lie au travail d'écriture et à l'avidité de voir et qu'elle retrouve ailleurs, dans Orion aveugle ou La Route des Flandres. Tel encore celui de l'animalité, qui réfere à la diversité des espèces du Jardin des Plantes, animalité énigmatique ou inquiétante, prise dans les oppositions de la violence prédatrice mais aussi, pour Anne-Lise Blanc, comparant pour dire l'indicible de la peur (Blanc 1999). Sa réflexion rejoint l'étude de Els Jongeneel soulignant l'animalité de l'homme pris dans la guerre (2001). Annie Clément-Perrier (1999) étudie la fonction poétique des couleurs, 
leurs effets de contraste et de leitmotive, leur pouvoir émotionnel et les polarisations affectives qu'elles révèlent. Nathalie Roelens note l'ambivalence de motifs tels que le calice et la fleur, associés au thème de l'eau - euphoriques ou dysphoriques selon une oscillation familière aux lecteurs de Simon; ce qui l'amène à ouvrir le texte sur la peinture et à voir dans Le Jardin des Plantes une sorte de " gigantesque ekphrasis romancée du Triptyque des délices de Bosch " (Roelens 2001: 79), rejoignant ainsi les études sur les relations entre écriture et peinture.

\section{INTERTEXTUALITÉS}

Caractérisé par une polyphonie extrême, Le Jardin des Plantes invite à l'étude de toutes les formes d'intertextualité, depuis la réécriture de romans antérieurs jusqu'aux relations entre les romans et le cinéma, en passant par l'intertextualité, par la métatextualité et même les études génétiques si l'on veut bien considérer que, donnant à lire le travail de reformulation, celles-ci sont proches de l'étude des réécritures.

\section{Réécritures}

Partons des études consacrées à celles-ci, si caractéristiques d'une œuvre qui revient sans cesse sur ses propres traces pour, obstinément, tenter d'autres formulations. Partant de l'enregistrement de l'auteur dans les épisodes avec le journaliste, Didier Alexandre, dans une analyse phénoménologique de la voix et de la répétition, voit le magnétophone comme le symbole du mouvement de l'œuvre " tournée vers l'arrière pour mieux progresser vers l'avant" (Alexandre 1999: 8). Car la répétition qu'il représente rend audible ou visible la nécessité d'une réécriture ne cessant $d$ ' "inachever " l'œuvre. Cet article très riche du premier recueil collectif consacré au Jardin des Plantes ouvre de nombreuses pistes: le privilège accordé à la diction et à la singularité d'une voix sur la fiction, le statut générique paradoxal du livre, la recherche de l'originaire, le lien entre rumeur et mélancolie, l'indépendance de l'écrivain; pistes qui seront reprises et poursuivies par d'autres. C'est aussi en termes de sonorités que Patrick Longuet parle des échos des romans antérieurs venant inscrire, dans Le Jardin des Plantes, "une manière de bruit" (Longuet 2000: 26) : quelque chose d'insistant et d'incertain, une « rumeur ». Et ressortent, sur le fond de cette rumeur, des révélations, des scènes d'enfance et d'humiliation qui, elles, échappent à la reprise et à la réécriture. Lors du même colloque, le premier consacré au roman, en 1999, J.-Y. Laurichesse s'arrête sur les nombreuses réécritures qu'opère celui-ci 
d'Album d'un amateur (Laurichesse 2000) : de l'avidité du regard naît la multiplicité des choses vues, un éclatement géographique qui fait de chaque fragment une île dans ce " récit de voyage en archipel " (ibid.: 126). Le Jardin des Plantes est l'album d'un voyageur, mais un album sans images qui rend hommage à la description. Quand le même critique étudie la réécriture, plus de quarante ans après, du quatrième chapitre du Sacre du printemps (Laurichesse 2002), il lit celui-ci comme moment d'un apprentissage de l'Histoire loin de tout héroïsme. Alors que ce roman racontait, en un autoportrait cruel, l'initiation à un monde de violence primitive, Le Jardin des Plantes et sa suite discontinue de fragments gomment toute dramatisation dans une sorte de mise à nu de l'épisode. Seule la figure du Comandante, inaltérée, affiche la même énigmatique ambiguïté.

Parmi ces échos des romans antérieurs, la réécriture de l'expérience traumatique fondatrice du printemps 1940 occupe une place majeure et plusieurs articles lui sont consacrés. Corinne Grenouillet (2000) étudie d'un point de vue stylistique la reprise des formulations de La Route des Flandres dans Le Jardin des Plantes et le double mouvement de condensation narrative et d'expansion relative, témoin du caractère inépuisable du référent pour Simon. Dans l'article cité, M. Calle-Gruber interprète le leitmotiv de la scène du printemps 40 comme une "déconstruction" de La Route des Flandres "pour une immense recomposition à l'échelle du siècle et du monde "(Calle-Gruber 2000: 54). Renée Ventresque, elle, recherche méthodiquement parmi les variations opérées sur la scène de la mort du colonel, la singularité de celle qu'offre Le Jardin des Plantes (Ventresque 2000) : une scène qui amène à conclure, malgré les documents nombreux reproduits, à l'instabilité et à la relativité de tout récit. Seule l'image du colonel et de son cheval tombant maintient ses contours, fixés par le vitrail vu dans l'enfance. La critique voit également dans l'aveu de S., reconnaissant avoir accepté d'être " assassiné » en acceptant de suivre son colonel, la possibilité de la fin du ressassement. Les études des réécritures opposent ainsi la permanence quasi rétinienne des images mémorielles à l'instabilité des autres souvenirs et de leur mise en mots.

\section{Intertextualité}

L'intertextualité du Jardin des Plantes est généralisée et polymorphe, comprenant aussi bien des textes littéraires que des documents hétérogènes. Les lecteurs de Simon connaissent la relation étroite qu'il entretient avec certains auteurs, Dostoïevski, Proust et Conrad en particulier, mais ceux-ci, jusqu'alors 
présents discrètement, cités ou récrits, le sont ici ouvertement et nommément, par l'enchâssement de citations dans le texte.

Parmi les nombreuses références à Dostoïevski, déjà commentées par J. H. Duffy (1998), Anne-Yvonne Julien revient sur la citation d'un dialogue entre deux " démons ", un théoricien du suicide et un nihiliste: cette « histoire de feuilles» (Julien 2006) et de temps suspendu s'inscrit en contrepoint du regard avide de matières et de détails que porte sur une feuille tournoyante le voyeur du balcon, aussi narrateur-descripteur. Sans doute Simon refuse-t-il la réponse métaphysique de Dostoïevski, cependant Cécile Yapaudjian-Labat propose de voir en celui-ci une autorité qui permettrait d'introduire des questions comme celle du sujet et de l'existence de Dieu; à l'arrière-plan de la mélancolie, se perçoit ainsi l'affirmation "d'un nihil humain face à la transcendance divine, la proclamation d'un nibil duquel surgit le malheur de la conscience séparée " (YapaudjianLabat 2011 : 192). Dans la continuité des articles de Marie Miguet-Ollagnier ${ }^{9}$, des recherches récentes s'intéressent à l'intertexte proustien pour croiser intertextualité et poétique compositionnelle: Chiara Falangola (2010) analyse ainsi le jeu des reprises et échos entre les fragments successifs dans la construction d'un récit fragmentaire et hétérogène, selon une sorte de simultanéisme narratif. Dans le dernier chapitre de son ouvrage, De Proust à Simon: le miroitement des textes, Laurence Cadet souligne l'écart des citations proustiennes du Jardin des Plantes avec le détournement parodique de La Bataille de Pharsale, et observe un devenir " miroir » et "objet fantasmatique " de Proust pour Simon qui le réécrit (Cadet 2011: 284 et 296). Christine Genin voit également Simon prolongeant les recherches proustiennes: "Simon a si bien lu et admiré le texte de Proust qu'il applique et améliore le procédé de contrepoint [...] afin de faire coexister deux moments très différents " (Genin 2016: 129), en transposant la projection simultanée d'images différentes sur plusieurs écrans ${ }^{10}$. Outre ces deux figures tutélaires souvent et régulièrement invoquées par Simon ${ }^{11}$, on sait que le nom de Joseph Conrad, récurrent dans les références et les entretiens, apparaît en exergue au chapitre III. Emmanuel Bouju voit dans la «boucle épigraphique » (Bouju 2007) formée par l'exergue une téléologie tenue à distance critique: la citation n'est plus

9. M. Miguet-Ollagnier, "Le Jardin des Plantes à l'ombre de Marcel Proust ", Bulletin d'Informations Proustiennes, no 29, 1998, p. 129-140; et "Claude Simon à l'ombre du Jardin des Plantes", dans B. Degott et M. Miguet-Ollagnier (dir.), Écritures de soi: secrets et réticences, L'Harmattan, 2001, p. 351.

10. Selon les mots de Claude Simon dans JP, p. 1055.

11. Lors d'un entretien avec Christian Michel et Richard Robert, Simon déclare: «Je place Dostoïevski audessus de Proust qu'en dépit de quelques réserves je place lui-même très haut. Toutefois, on n'a, à mes yeux, jamais poussé aussi loin ni aussi haut l'art du roman et celui de la prose que Conrad ne l'a fait avec ces deux chefs d'œuvre d'orfevvrerie que sont Typhon et Le Nègre du Narcisse » (Scherzo, nº 3, avril-juin 1998). 
un argument d'autorité, le texte reconnaît ainsi dépendre d'un questionnement plus large et ancien, le "questionnement même de la littérature ", une façon subtile de faire sens avec un intertexte en le " vaporisant ».

Parmi les autres écrivains présents mais de manière allusive, figurent Montesquieu et Borges, intertextes respectivement analysés par Anne-Yvonne Julien et Katerine Gosselin. A-Y. Julien (2007) relève les allusions aux Lettres persanes, nombreuses dans le contexte satirique du voyage en URSS, comme participant d'un regard et d'une impulsion dialogique puisés dans le voyage. Partant de la relecture de Stendhal dans l'entretien avec le journaliste, K. Gosselin (2015) attribue une fonction matricielle au récit de la bataille de Waterloo dans La Chartreuse de Parme pour faire observer la lecture contradictoire qui en est faite, biographique et néo-romanesque. Mary Orr (2002) propose de son côté une étude très argumentée des liens intertextuels du Jardin des Plantes avec Le Jardin aux sentiers qui bifurquent de Borges et élargit son propos aux stratégies intertextuelles neuves proposées par le roman: le dialogue instauré entre Proust et Flaubert caractérise l'écriture comme travail, dans un intertexte fait de matériaux d'écriture jusqu'ici disponibles seulement pour les chercheurs. M. Orr voit enfin dans Le Jardin des Plantes une exploration particulièrement complexe et claire de ce qui distingue $S$. de Claude Simon et une «somme des préoccupations intertextuelles et des réécritures de $\operatorname{Simon}^{12}$ ».

\section{Génétique}

$\mathrm{Si}$, outre la réédition de prépublications, plusieurs composantes de la genèse du Jardin des Plantes ont été publiées - un plan de travail (2008), des pages manuscrites ${ }^{13}$ - les études génétiques de ce roman en sont encore à leurs débuts. J. H. Duffy (2006: 1467), M. Calle-Gruber (2011 : 416-17) puis Isabelle Diu et Sophie Lisiewicz (2014: 166) ont décrit le dispositif mis au point par Simon pour les plans d'assemblage: des languettes de papier numérotées et amovibles sur des cadres-plans qui produisent une vision simultanée des diffé-

12. "S. and Claude Simon are also not the same. Simon's exploration of how they are not and how they can be distinguished are demonstrated with a new complexity and a new clarity in Le Jardin des Plantes"; "summa of Simon's intertextual preoccupations and "réécritures" (Orr 2002: 133).

13. Le fonds Claude Simon, qui avait fait l'objet d'une donation en 1984, est entré à la Bibliothèque littéraire Jacques Doucet après la disparition de l'auteur entre 2006 et 2007. Voir le témoignage d'Yves Peyré dans ce numéro (p. 27-29); voir également sa description par Isabelle Diu et Sophie Lisiewicz (2014). D'autres manuscrits sont publiés sur le site de l'Association des Lecteurs de Claude Simon (notamment la fiche d'une citation de Dostoïevski à l'adresse [http://associationclaudesimon.org]) et sur le blog de l'ARCS (Archive: Claude Simon et ses contemporains, [http://arcs.hypotheses.org/499]). 
rentes séquences. Les prépublications du roman ont fait l'objet de rééditions ${ }^{14}$ : "Les Jardins publics", "Lecture publique d'une pièce de théâtre. Fragment d'un texte " et "Polygone ". Bien qu'on ne puisse parler de prépublication à son propos, c'est bien un avant-texte du Jardin des Plantes que réédite en 2006 J.-Y. Laurichesse: "Cendre ». Ce texte de 1959, paru dans la très classique Revue de Paris peu avant La Route des Flandres, est le récit d'une longue hospitalisation dont on trouve de nombreux motifs dispersés et réécrits dans les parties I et III de notre roman. J.-Y. Laurichesse (2006) éclaire les liens multiples tissés avec le contexte qui donnent un surcroît de sens à cette expérience, devenue fondatrice, à l'égal de celle du 17 mai 1940. Un seul article de critique génétique porte sur le manuscrit du Jardin des Plantes, celui d'Olivier Kahn (2016) : à partir des premiers feuillets, celui-ci montre que loin de l'image qu'on se fait d'un écrivain travaillant sans plan préconçu, et pas à pas, les manuscrits de Simon présentent des sous-plans, des listes: rédaction et programmation constituent donc tout au long le travail d'écriture.

\section{Métatextualité}

L'étude des commentaires métatextuels s'impose dans Le Jardin des Plantes dès le titre et les exergues qui font du jardin parisien une figure de sa composition. Ce «roman» qui rassemble des «fragments épars d'une poétique du roman " est décrit comme une fiction critique par Brigitte Ferrato-Combe (2002: 145); il intègre une critique du langage et du cliché, et fictionnalise des éléments de paratexte: il diabolise l'œuvre de l'auteur en rappelant la condamnation prononcée à son encontre à Cerisy, et renforce ainsi la posture provocatrice de l'écrivain. P. Dirkx et P. Mougin (2011) de même, introduisant au volume collectif Situations, décrivent Le Jardin des Plantes comme le « condensé esthétique " de l'entreprise romanesque de Simon, un métadiscours en acte sur sa poétique. Ces réflexions sur la métatextualité sont désormais bien loin des débats autour de l'autonomie de l'œuvre de langage et ne gardent plus trace de ce que pouvaient avoir d'antagonique, dans les années 70, les notions d'autoréférentialité et de référence. Nul ne voit plus d'incompatibilité entre les études concernant les mises en abyme et celle d'un personnage historique comme Gastone Novelli, plus ou moins fictionnalisé. Cependant le point de vue adopté peut, selon les cas, rester interne à l'œuvre, envisager sa relation au contexte ou bien passer de l'un à l'autre.

14. Voir la bibliographie ci-dessous. 
J.-Y. Laurichesse (2001) analyse la mise en abyme que constitue l'épisode amazonien de Novelli, récit singulier en ce qu'il s'inscrit dans une causalité et une chronologie: s'y jouent aussi bien le rétablissement d'une relation à autrui après l'expérience du camp nazi que le développement de l'œuvre à venir. Laurichesse voit en Novelli un double tragique du romancier en même temps qu'un avatar de la figure mythologique d'Orion. D'autre part, de façon plus interne, le même critique étudie trois fragments descriptifs de ruines et il éclaire la capacité de l'écriture fragmentaire à " inscrire dans son contenu même sa propre métaphore " (Laurichesse 2002: 291) ; les ruines sont dotées de fonction métapoétique: arracher le fragment au néant. De même, pour A.-Y. Julien (2007) qui s'intéresse aux " archipels d'îlots " des pourtours urbains, le roman explore toutes les variantes de l'écrit spéculaire.

Cependant la part la plus manifeste de métatextualité tient à la présence récurrente de l'auteur, qu'il soit en première ou troisième personne, "je " ou S., l'auteur devenant personnage d'écrivain. Au colloque de 1999, à Perpignan, Dominique Viart met en lumière ce retour critique et ironique de Simon sur son ouvre et sur sa posture d'écrivain: "Moins que jamais l'auteur y est une autorité» (Viart 2000: 10). Le critique montre Simon jetant le trouble entre les catégories de fiction et de réel, et précise que ce "roman » énonce bien du réel mais du « réel représenté, c'est-à-dire la fiction mentale et verbale que l'écriture traduit du réel » (ibid.: 13): un "roman » qui n'élude "rien des questions que pose l'acte d'écrire " (ibid. : 23), jusqu'à l'exploitation idéologique dont est menacé un prix Nobel. L'auteur se laisse donc approcher plus que jamais - avec ses référents, ses autobiographèmes - et Cora Reitsma-La Brujeere (2001) note le rôle de médiateur que joue le journaliste, tandis que Katerine Gosselin (2015) observe le déplacement de l'opposition faite d'ordinaire entre le faire et le dire de l'écrivain: Simon intègre au roman le langage balbutiant de l'auteur sur ses livres et ses silences. Sur la question du genre, ce n'est pas un modèle littéraire que Patrick Suter (2009) voit contesté dans Le Jardin des Plantes mais davantage et en référence à Mallarmé, l'écriture journalistique, par le refus de la " colle » de la causalité et l'usage du fragment.

\section{Relations intersémiotiques}

Une dernière forme d'intertextualité concerne les relations du texte - que l'on dira intersémiotiques - à ses références picturales ou artistiques: Véronique Gaultier (2005) réunit ainsi dans une même étude de l'iconographie les références à la peinture, à la sculpture et la photographie. L'analyse peut considérer 
l'œuvre picturale ou cinématographique dans sa fonction interne au texte ou bien aussi en elle-même. Brigitte Ferrato-Combe (2000) rapproche la mise en forme du roman et la réflexion qu'il induit sur le langage de la "composition en damier " des toiles de Novelli et sa tentative de retour à une langue de l'élémentaire. M. Calle-Gruber la rejoint quand elle lit les « pavés de A » du peintre italien comme une "sorte de scène primitive de l'être-au-monde " (Calle-Gruber 2004 : 147). Un autre article de B. Ferrato-Combe sur Poussin s'interroge plus largement sur les significations prêtées à "La Peste d'Asdod" dans le cadre d'une réhabilitation du classicisme (Ferrato-Combe: 2001); le tableau répond indirectement à la question: comment vivre avec la peur, et invite à lier le point de vue des spectateurs du balcon au regard à distance de Simon - curieux du monde, voyeur à sa fenêtre. Le tableau de Poussin retient aussi l'attention de Jean H. Duffy (2002) en ce qu'il s'intègre à une méditation sur la foi et la relativité historique et géographique des divinités, ainsi que sur les aléas de la communication; la critique souligne elle aussi le choix du retrait de l'écrivain et la recherche d'une cohérence esthétique venue, pour Novelli comme pour Poussin, non de la biographie mais d'échos formels et chromatiques.

Malgré les références fréquentes qu'il fait au cinéma, et malgré le scénario final, il existe peu d'études intersémiotiques sur Le Jardin des Plantes. Deux analyses l'éclairent de manière très différente; celle de Sophie Charlin (2007) lit le montage à la lumière des théories de Vertov, de Eisenstein et de la notion de "résidu d'image ". Notant que l'intervalle chez Simon ménage des arrêts tout en servant de corrélation visuelle, S. Charlin souligne la forte discontinuité du montage disparate de la fin, où la primauté de la série "marche vers la mort du colonel " se défait progressivement. Ce même scénario est mis en perspective avec la création simonienne par Bérénice Bonhomme. Contrairement à un premier découpage destiné à un projet de film sur La Route des Flandres, il n'est destiné à aucune mise en image mais doit se lire en relation avec les textes. C'est l'échec cuisant de ce premier projet, auquel Simon tenait beaucoup, qui « soustend " le découpage du Jardin des Plantes (Bonhomme 2010 : 384) marqué par la distance et l'ironie: grotesque des images, montage burlesque, accentuation presque caricaturale des mises en abyme.

\section{RÉFÉRENTS ET RÉFÉRENCE}

Les études qui se donnent pour objet les rapports du roman au « réel » ou à son contexte soulignent toutes la tension entre la visée impossible de dire 
le " réel » ou le " vécu » et les exigences d'une écriture dans et par la langue. Le Jardin des Plantes leur est un terrain propice puisque Simon y assure, selon les mots de P. Dirkx et P. Mougin (2011: 22) «l'assomption du référent comme présence contextuelle dans un texte qui néanmoins la transfigure ».

Très tôt, la critique a souligné la distance prise avec le genre autobiographique, dans ce livre qui manifeste visuellement avec force le morcellement du sujet et l'impossible achèvement d'une écriture de soi. Ce morcellement tient pour Dominique Viart (1998) à la figuration de l'écrivain " en situation » qui reprend inlassablement les motifs mémoriels des livres antérieurs en tentant de cerner "l'indicible». Jean H. Duffy, pour sa part, précise les traits qui empêchent de lire ce "roman " comme une autobiographie: Simon, de façon multiple et toujours indirecte, au travers d'éléments biographiques d'artistes et d'écrivains, répète qu'il est impossible de dire son expérience (Duffy 1998). L'écriture de soi est à la fois inévitable et inaccessible, tension entre expression et silence, entre assemblage et morcellement, entre visée d'une cohérence et refus de clôture. Quand Alastair Duncan, en 2002, complète son ouvrage Adventures in Words pour intégrer les deux derniers romans, il souligne, dans Le Jardin des Plantes, le renouvellement qui consiste à donner une forme visuelle à la simultanéité de la mémoire, et à dire son expérience dans un langage "non linéaire, indirect, médié par la fiction" (Duncan 2002: 156). Le paradoxe de ce portrait d'une mémoire, pour Patrick Longuet, tient en effet à ce que, de cet assemblage d'extraits, de bribes de fictions, de Mémoires, de textes, " un roman s'impose ", " un lieu d'intimité conciliée où l'art naît d'un travail " (Longuet 2015: 12).

Dans une perspective psychanalytique, Stéphane Darnat s'attache à la voix problématique qui, " entre le Je et le Il [...] hante l'espace fragmentaire » (1999: 19); il analyse le traumatisme du 17 mai 40 comme objet d'un deuil impossible, promis au ressassement, ressassement dont il lit une figure dans l'écran noir des dernières pages. Partant de la fascination de l'enfant pour une gravure de vestale ensevelie, Pascal Mougin montre qu'entre Le Tricheur et notre roman, s'opère un renversement du travail du deuil de la mère, et avec l'hommage silencieux à celle-ci, une phobie de l'enlisement, de " la peur dans le fond " mais tenue à distance. Notant que, pour la première fois, l'épreuve de la mort est présentée comme "relevant du libre arbitre ", le critique y voit une "mort initiatique ", et l'occasion d'une renaissance (Mougin 2000: 98). Rejoignant A. Duncan (2002) qui lit les différentes formes de contextualisation comme atténuant l'aiguillon du trauma de 1940, Celia Britton (2002) revient sur cette scène dite " traumatique " par Simon lui-même, pour montrer que le 
texte en neutralise la violence en l'intégrant à d'autres souvenirs et en l'insérant dans la durée et la répétition. Dans une lecture plus lacanienne que freudienne, Llewellyn Brown étudie, à travers les motifs du voile et de l'eau, puis du motif phallique du A majuscule, comment «la représentation se fonde sur ce qui la dénie, voile l'innommable " pour y lire «l'équivoque fondamentale de tout langage " (2012: 255), au risque d'y manquer la spécificité de ce livre, écrit en grande partie par d'autres.

Parmi les études qui abordent de front la question de la référence au monde, deux sont issues de la recherche anglo-saxonne, pionnière en la matière, celles de David G. Ellison et de Jean H. Duffy. Partant de la description du Jardin des Plantes parisien et des références à Proust et Rommel, le premier montre combien « le réel est un point de départ pour le fictionnel " (Ellison 2002: 138) et suit le fil de l'opposition nature/culture, en particulier dans la seconde partie qui oppose la destruction de la guerre à la conversation mondaine et esthétique. Simon fait ainsi de la littérature " un lieu à partir duquel le combat des objectifs réciproques de la nature et de la culture puisse être productif ${ }^{15}$ ". L'étude de J. H. Duffy citée plus haut (2002: 189) est aussi significative de la complexité qu'exige le traitement du référent, non plus littéraire mais pictural, montrant dans le tableau de Poussin le support d'une double réflexion sur l'écriture et sur la foi comme besoin permanent de donner du sens malgré la relativité des croyances. Dans ces deux études, c'est le travail opéré par Simon à partir du référent (textuel ou pictural) qui est analysé et les effets de sens qui en découlent. C'est aussi le cas de la lecture proposée par Bertrand Westphal (2013) qui voit le Jardin des Plantes parisien comme le centre vide d'une forme d'autobiographie dont la dynamique géographique dépend de l'agencement et de l'interaction des différents lieux.

La question du personnage référentiel était sensible pour Simon ${ }^{16}$, et l'étude de Didier Alexandre (2000) sur Rommel et Churchill éclaire la tension entre historiographie et littérature. Le critique analyse la transposition des matériaux que sont les carnets de Rommel, les archives militaires et les mémoires de Churchill: comment celui-ci est identifié à son icône et celui-là à sa trajectoire

15. " un lieu à partir duquel the reciprocal claims of culture upon nature and of nature upon culture engage in the only kind of war to which the adjective "productive" can be adjoined" (Ellison 2002: 151; nous traduisons).

16. En octobre 2002, dans une "Note sur Le Vent" envoyée aux éditeurs de son ouvre dans la "Pléiade", Simon rappelle les raisons de l'anonymat des lieux et personnages et met en garde contre toute référence à un " modèle ": " les personnages ne doivent leur existence (ou plutôt leur matière) qu’à l'écriture ellemême" (EI, p. 1203). 
militaire, devenant «l'agent du désastre ${ }^{17}$ " (Alexandre 2000: 85). Cette tension productrice a permis l'entrée dans le champ de la critique simonienne de la notion d'archive empruntée aux historiens, entrée décisive dont témoignent le volume dirigé par Ralph Sarkonak, Le (Dé)gồt de l'archive, et l'article de Jacques Isolery qui en démontre le rôle et l'enjeu fondateurs. Caractérisant le " roman " comme une collection d'archives, celui-ci les compare aux ruines en ce qu'elles donnent au fragment toute sa puissance évocatoire de multiplicité et de diversité et incarnent "la résistance même de la vie " (Isolery 2005: 60). À côté de l'enjeu esthétique - organiser des rapports de résonances -, le critique souligne l'enjeu éthique: en intégrant ainsi la présence d'autrui, l'œuvre d'art se distingue clairement de l'historiographie et la diversité des archives défait l'illusion d'un accès possible à leur vérité. Michèle Touret, observant l'écriture de la guerre et la mise en scène de la pluralité des points de vue et des rôles, s'inscrit dans la continuité de ces lectures. Le document ne peut être attestation de vérité et dès lors, la littérature "crée les conditions de la critique de toute lecture et de sa propre lecture " (Touret 2011: 158).

On sait que Simon s'est toujours opposé à tout engagement de la littérature et qu'il affirmait volontiers n'avoir "rien à dire ". Mais ses livres? Leur lecture peut se faire sans tenir compte des intentions déclarées de l'auteur. La critique de l'engagement est, pour Nathalie Piégay-Gros, «le résultat du soupçon radical porté sur le récit ", une conclusion tirée des apories de la littérature qui relève "d'un point de vue éthique au moins autant qu'esthétique » (2005: 285). Son étude du montage comme sapant toute prétention à l'objectivité conclut à une " mélancolie du récit " (ibid.); elle transpose ainsi à sa poétique la mélancolie simonienne, avidité et lucidité extrêmes. J.-Y. Laurichesse (2005) relève lui aussi le défi de confronter la pratique d'écriture de Simon à ses principes théoriques: le roman ne cherche pas à énoncer de vérité, mais n’en dit pas moins, indirectement, par le biais des situations et des descriptions, le « drame de la perte du sens " (ibid.: 4). Il critique certaines valeurs au nom d'autres valeurs, comme celle qu'incarne Novelli. Cette dimension éthique est également soulignée par S. Houppermans (2001) qui, notant ce qui rapproche Simon et Beckett, comme la certitude de la solitude radicale du sujet, montre le lien entre art poétique, position historique et éthique: dépouillement de Beckett, "droit de dire non » de Simon (ibid.: 142). Enfin la portée critique du Jardin des Plantes a fait l'objet de quelques articles: la force satirique de la gouaille a

17. Dans « La Critique simonienne et le référent: l'insistance du réel », J.-Y. Laurichesse décrit la complexité de la position de Simon sur cette question (Laurichesse 2012). 
intéressé Maurice Roelens qui en souligne le " "tonus" polémique et critique " (Roelens 2000: 66), tandis que Metka Zupancic observe un phénomène de désacralisation généralisée (Zupancic 2001). R. Sarkonak pour sa part, en relève l'ironie: dans la charge contre le dogmatisme formaliste, "le refus de l'archive [...] devient matière d'une nouvelle archive, cette fois-ci de la bêtise d'un savoir qui se voulait universitaire" (Sarkonak 2005: 18).

Parallèlement, la critique simonienne s'est emparée de ce que le roman dit de son contexte et en particulier de la situation et de la position de l'écrivain dans le monde, déplaçant ainsi la question de la métatextualité. Après avoir suivi les réincarnations textuelles de l'écrivain en tant qu'écrivain jusqu'au Jardin des Plantes, l'étude de P. Dirkx (2012) voit dans ce roman une analyse en acte de sa position littéraire d'écrivain solitaire: radicalisation de l'inventivité et dénonciation indirecte des censures. L'initiale "S. " souligne la nature différente de la réalité et de la fiction, pourtant intimement liées et appartenant au même monde. Le colloque "Claude Simon à la lumière de l'histoire littéraire, de l'histoire culturelle et de la sociologie de la littérature ", organisé par P. Dirkx et P. Mougin en 2008 et publié sous le titre Claude Simon: situations, prend acte des mutations de la critique simonienne et privilégie les approches qui se placent dans un au-delà du texte. Ces études ne sont pas pour autant sociologiques : elles considèrent le référent comme "élément actif interne " et le texte "comme produisant sa propre réalité » (Dirkx et Mougin 2011: 16). Après une analyse de l'insistance sur le référent comme réponse polémique au formalisme néoromanesque, Yona Hanhart-Marmor (2011 : 165) montre que la correspondance établie entre la situation solitaire de l'écrivain et son écriture (fragmentaire, difficile) "conduit à admettre qu'on ne peut pas séparer l'écrivain dans le monde de l'écrivain devant sa table de travail » et à lier "l'exigence extrême " de l'écriture simonienne à son refus de "se scinder en écrivain et homme public, ou écrivain et témoin, ou écrivain et théoricien " (ibid.: 178).

Si Claude Simon n’a "rien à dire " au sens sartrien, les significations multiples et les effets de montage des fragments laissent donc une grande liberté d'interprétation. Ainsi le montage peut-il être considéré comme un facteur d'illisibilité en ce qu'il est « retrait du discours » selon N. Piégay-Gros (2005), ou bien comme incitation, invitation au lecteur, selon Joëlle Gleize (2015), qui fait l'hypothèse d'une transformation de la lecture inscrite dans les deux derniers romans: comparant la lecture souhaitée par l'auteur dans ses conférences avec les figures de lecteur et les nombreux repères et signaux fournis par le texte (références, usage du blanc typographique), l'étude souligne la préé- 
minence nouvelle d'une adhésion sensible sur une lecture des harmoniques. M. Touret (2011) pour sa part, voit dans l'usage des matériaux documentaires une sorte de "leçon " indirecte sur la littérature: leur fonction doit être plutôt cherchée du côté du lecteur avec lequel il s'agit d'établir une collaboration d'ordre littéraire. Cette considération du lecteur rejoint l'analyse déjà évoquée de J. Isolery: «En reconfigurant les archives dans des collections instables dont la diversité invite à autant de lectures tabulaires [...], l'écriture simonienne interdit leur rabattement sur une interprétation et une signification univoques " (Isolery 2005: 94-95); ou encore celle de Claro, pour qui «la multiplicité des images qui pourrait (et peut) produire de la confusion, crée avant tout du mouvement et de l'organique: d'innombrables entrées sont possibles au lecteur» (Claro 2015: 25). D’une de ces entrées multiples possibles témoigne P. Dirkx (2015), qui fait une lecture politique de la description érotique, manière de proclamer l'indépendance de l'écrivain et de défier les pouvoirs. Centrée sur le "foyer narratif » qu'est la scène de la salle de bains, son étude en souligne le caractère transgressif, et l'association, jusque dans la mise en page, de la peur de la mort et de son oubli dans le désir. C'est une lecture anthropologique que fait D. Viart de l'intérêt de Simon pour les rebuts, déchets et graffiti: à ses yeux ces matériaux du bricolage, "signes élémentaires de l'humain ", manifestent moins une mélancolie qu’une " humanité essentielle, mesurée à ses manifestations premières: la peur, le désir sexuel, la menace de mort et la pulsion vitale " (Viart 2015: 116). À la diversité textuelle répond ainsi la diversité des lectures, plus que jamais ouvertes.

Autre manifestation d'ouverture et de liberté prise par le lecteur, nombre de travaux envisagent l'écriture de Simon dans le contexte de la littérature française ou européenne et opèrent des rapprochements. Ainsi S. Houppermans éclairant la parenté de Simon et Beckett: tous deux solitaires, ils remettent en cause, à travers leur histoire personnelle "le récit du monde et de l'univers ", ils redramatisent l'art "selon une ultime rencontre du plus matériel et du plus spirituel, "ecce homo" " (Houppermans 2001 : 137). Quant à E. Bouju, on l'a vu aussi, lisant l'épigraphe comme un hypertexte secret, il rapproche Simon de W. G. Sebald, et leur « auto-critique ultra-sceptique sur les moyens de la littérature face à l'histoire " (Bouju 2007). Le Jardin des Plantes est fortement présent dans Austerlitz ${ }^{18}$, où se retrouvent un rapport à la mémoire et au passé, une méfiance du récit chronologique proches de Simon ${ }^{19}$. La mise en contexte peut

18. W. G. Sebald, Austerlitz (tr. P. Charbonneau), Arles, Actes Sud, «Lettres allemandes ", 2002.

19. Sur le rapport au passé de C. Simon et W. G. Sebald, voir Tennstedt 2007. 
aussi souligner des différences: comme celle des formes et visées du montage chez Simon et Michel Leiris qui privilégie (dans la revue Documents) la fonction critique sur la visée esthétique (Gleize 2008). Pour Wolfram Nitsch (2013), qui observe les vues aériennes dans l'œuvre de Simon, replacer ces visions de la terre dans leur contexte culturel en souligne la violence latente sous l'abstraction et l'immobilité de la vue d'avion, et l'opposition à la vision héroïsante de l'aviateur - tel Saint-Exupéry vu par Sartre. Cécile Yapaudjan-Labat (2010: 434) souligne ce qui sépare Simon d'Alain Robbe-Grillet: si la ruine apparaît comme «le lieu du plaisir et du jeu » chez Robbe-Grillet, chez Simon, pour qui " désir et tragique de l'existence ne font qu'un », elle est le lieu d'une écriture du désir. Parmi les ouvrages qui placent l'œuvre de Simon dans le contexte d'après Auschwitz et de la faillite des " grands récits » explicatifs ${ }^{20}$, Sabrina Parent (2011), qui caractérise l'écriture de Simon par l'abandon d'une éthique de la conviction au profit d'une recherche d'authenticité, s'appuie sur l'exemple du Jardin des Plantes et du renoncement de S. à expliquer l'événement historique ("l'étrange défaite ») pour tenter de formuler son ressenti.

Dans ce "roman" simonien plus encore que dans d'autres peut-être, les entrées sont multiples autant que les cheminements critiques pour donner, en miroir de ce " portrait d'une mémoire " éclaté et diffracté, un ensemble de lectures à la fois divergent et cohérent, du moins sans divergences irréductibles. Portrait en « fragments épars » d'une lecture attentive à la singularité de ce livre, à tous les filtres et à toutes les images de la mémoire, au regard désirant porté sur le monde, depuis la feuille jusqu'à la terre vue du ciel, à la distance toujours tenue (et avec humour souvent), à la position de l'auteur, à la recherche tenace de la formulation la plus juste musicalement et par là même, la plus "vraie ".

"Et à la fin il dit Mélancolie» (EI, p. 1120), formule sans doute la plus souvent citée, et mot carrefour, qui clôt et relance le discours critique comme il relance le texte, puisque l'auteur corrige aussitôt les visions suscitées: le dernier mot n'est jamais le dernier.

20. Nombre de doctorats en cours portent sur un corpus où Claude Simon est associé à d'autres écrivains ou courants littéraires contemporains, et étudient le contexte culturel de son écriture ou sa postérité (contestation anti-épique, primitivisme, par exemple). 


\section{LE JARDIN DES PLANTES UNE BIBLIOGRAPHIE CRITIQUE ET CHRONOLOGIQUE}

\section{TEXTES DE CLAUDE SIMON, AVANT LE JARDIN DES PLANTES}

"Cendre ", La Revue de Paris, mars 1959, p. 79-82 (repris dans Cahiers Claude Simon, $\mathrm{n}^{\circ} 2,2006$, p. 133-137).

"Les Jardins publics », dans Mireille Calle-Gruber (dir.), Les Sites de l'écriture: Colloque Claude Simon, Nizet, 1995, p. 25-37.

"Lecture publique d'une pièce de théâtre. Fragment d'un texte ", dans L'Infini, n 56, hiver 1996, p. 3-10.

"Polygone", dans Cahiers de la Bibliothèque littéraire Jacques Doucet, n 1, 1997, p. 11-15.

«Le poisson cathédrale " (1980), Quatre conférences, Minuit, 2012, p. 9-38.

\section{ARTICLES ET OUVRAGES CRITIQUES}

1997

André Clavel, "Claude Simon, côté jardin ", Journal de Genève et Gazette de Lausanne, 20-21 sept. 1997.

Jean-Louis Ézine, "Claude Simon repart en guerre ", Le Nouvel Observateur, n 1715, 18-24 sept. 1997, p. 112 (v. aussi p. 110-116, le dossier sur le Nouveau Roman avec les « Propos » d'Alain Robbe-Grillet recueillis par J.-L. Ézine, où sont évoquées les pages du Jardin des Plantes concernant le colloque de Cerisy).

Fabrice Gabriel, "Allegro vivace », Les Inrockuptibles, 17-23 sept. 1997, p. 8-9.

Jacques Pécheur, "Simon, Le Jardin des Plantes», Le Français dans le monde, n² 293, nov.- déc. 1997, p. 16-17.

Isabelle Ruf, «Un jardin pour mémoire », L'Hebdo, 18 sept. 1997.

Thiphaine Samoyault, "Jardinier de la mémoire", La Quinzaine littéraire, n 724, 1-15 oct. 1997 , p. 5-6.

\section{8}

Annie Clément-Perrier, "Regard sur un motif du Jardin des Plantes ", Poétique, n 115, sept. 1998, p. 273-285. 
Jean H. Duffy, " "Ce n'est pas une allégorie. C'est une feuille tout simplement" : text, intertext and biography in Claude Simon's Jardin des Plantes", Romanic Review, $n^{\circ}$ 89(4), 1998, p. 583-607 (texte traduit et repris dans ce numéro, p. 111-137)

Michelle Labbé, « Paysages perdus dans Le Jardin des Plantes de Claude Simon », Courrier du Centre International d'Études Poétiques, n² 217, jan.-mars 1998, p. 21-37.

Marie Miguet-Ollagnier, "Le Jardin des Plantes à l'ombre de Marcel Proust », Bulletin d'informations proustiennes, $\mathrm{n}^{\circ} 29,1998$, p. 129-140.

Dominique Viart, "Remembrance et remembrement: cultiver les friches de la mémoire. Le Jardin des Plantes ", Scherzo, n 3, avril-juin 1998, p. 23-29.

\section{9}

Didier Alexandre, "L'enregistrement du Jardin des Plantes ", Littératures, n 40, 1999, p. 5-18 (repris dans ce numéro, p. 95-109).

Michel Bernard, "Le Jardin des Plantes ou l'hypotypose de la place Monge ", Fabula, 1999, [http://www.fabula.org/colloques/frontieres/213.php].

Anne-Lise Blanc, "Le "Jardin zoologique" du Jardin des Plantes. Visite guidée", Littératures, $\mathrm{n}^{\circ}$ 40, 1999, p. 47-57.

Mireille Calle-Gruber, "Claude Simon: Dans l'arc du livre il y a toute la corde » (entretien), suivi de "Claude Simon, un jardin de la mémoire où cultiver l'art d'écrire ", Nuit Blanche, $\mathrm{n}^{\circ}$ 74, printemps 1999, p. 55-60.

Annie Clément-Perrier, "Le jeu des couleurs dans le Jardin des Plantes ", Littératures, $n^{\circ} 40,1999$, p. 31-46.

Stéphane Darnat, "L'épiphanie du blanc », Littératures, n 40, 1999, p. 19-30.

Jean-Marc Dilettato, et Patrick Longuet, « Les feuilles au fond du bassin. Sur le Jardin des Plantes de Claude Simon ", L'Infini, n 65, printemps 1999, p. 88-111.

Jean H. Duffy, "Artistic biographies and aesthetic coherence in Claude Simon's Jardin des Plantes ", Forum For Modern Language Studies, n 35(2), 1999, p. 175-192.

Patrick Longuet, « "Et je pouvais voir”. Paysages, guerres culture, visages, corps, des flux et des restes: une lecture transversale de l'œuvre de Claude Simon ", ministère des Affaires étrangères (Espace culturel), 1999.

\section{0}

Didier Alexandre, "Le renard du jardin: remarques sur l'insertion du personnage historique dans le récit simonien ", dans J.-Y. Laurichesse (dir.), Cahiers de l'université de Perpignan, $\mathrm{n}^{\circ} 30$ ("Le Jardin des Plantes de Claude Simon, Actes du colloque de Perpignan [27 mars 1999] »), Perpignan, Presses universitaires de Perpignan, 2000, p. 67-88. 
Mireille Calle-Gruber, "Une harmonie contre tendue: des principes de l'arc et de la lyre appliqués à l'écriture du roman chez Claude Simon ", dans J.-Y. Laurichesse (dir.), op. cit., p. 39-56.

Brigitte Ferrato-Combe, "Simon et Novelli : l'image de la lettre ", dans J.-Y. Laurichesse (dir.), op. cit., p. 103-118.

Corinne Grenouillet, "Le Jardin des Plantes et La Route des Flandres, remarques sur la reformulation chez Claude Simon ", dans F. Migeot et J.-M. Viprey (dir.), Répétition, altération, reformulation dans les textes et dans les discours (colloque de Besançon, 2224 juin 1998), Besançon, Presses universitaires franc-comtoises, 2000, p. 113-130 (repris dans Semen, n 12, avril 2000, [https://semen.revues.org/1892]).

Michelle Labbé, "Les Paysages de l'esprit: La Bibliothèque, le jardin et la plage ", Études, février 2000, n 392(2), p. 235-243.

Jean-Yves Laurichesse, "Aux quatre coins du monde: Le Jardin des Plantes comme album d'un voyageur ", dans J.-Y. Laurichesse (dir.), op. cit., p. 119-136.

Patrick Longuet, "Echos et palimpseste. Sur Le Jardin des Plantes de Claude Simon ", dans J.-Y. Laurichesse (dir.), op. cit, p. 25-38.

Pascal Mougin, "Du Tricheur au Jardin des Plantes: la figure de la mère défunte ", dans J.-Y. Laurichesse (dir.), op. cit., p. 89-102.

Catherine Rannoux, "Éclats de mémoire: la page fragmentée, Le Jardin des Plantes de Claude Simon ", La Licorne, n 52, Poitiers, 2000, p. 245-260.

Maurice Roelens, "Figures de la "gouaille" et de la raillerie dans Le Jardin des Plantes ", dans J.-Y. Laurichesse (dir.), op. cit., p. 57-66.

Ralph Sarkonak, "Les Quatre Saisons au Jardin des plantes ", dans Revue des Lettres modernes, série "Claude Simon ", $\mathrm{n}^{\circ} 3$ ("Lectures de Histoire»), Caen, Minard, 2000, p. 191-214.

Dominique Viart, "Portrait de l'artiste en écrivain. Le Jardin des Plantes de Claude Simon ", dans J.-Y. Laurichesse (dir.), op. cit., p. 9-24.

\section{1}

Jan Baetens, "Éloge de la ligne ", dans S. Houppermans (dir.), Cahiers de recherches des instituts néerlandais de langue et de littérature (CRIN), $\mathrm{n}^{\circ} 39$ ("Claude Simon et Le Jardin des Plantes "), Amsterdam, Rodopi, 2001, p. 31-42.

Mireille Calle-Gruber, « Le récit de la description ou la nécessaire présence des demoiselles allemandes tenant chacune un oiseau dans les mains ", dans S. Houppermans (dir.), op. cit., p. 5-29.

Brigitte Ferrato-Combe, "Dans une lumière de Poussin ", dans S. Houppermans (dir.), op. cit., p. 119-131. 
Sjef Houppermans, "Claude Simon et Samuel Beckett: pour la mémoire du siècle ", dans S. Houppermans (dir.), op. cit., p. 133-145.

Els Jongeneel, "La ménagerie du Jardin des Plantes", dans S. Houppermans (dir.), op. cit., p. 109-117.

Jean-Yves Laurichesse, "Orion aveugle dans la forêt amazonienne. L'aventure de Novelli dans le Jardin des Plantes de Claude Simon ", dans S. Houppermans (dir.), op. cit., p. 45-61.

Marie Miguet-Ollagnier, "Claude Simon à l'ombre du Jardin des Plantes", dans Marie Miguet Ollagnier et Bertand Degott (dir.), Écritures de soi: secrets et réticences, L'Harmattan, 2001, p. 351-361.

Cora Reitsma-La Brujeere, "Vous permettez que j'enregistre?" L'interview dans le Jardin des Plantes ", dans S. Houppermans (dir.), op. cit., p. 63-72.

Nathalie Roelens, "Le Jardin des délices/supplices de Claude Simon ", dans S. Houppermans (dir.), op. cit., p. 75-86.

Renée Ventresque, "Le Jardin des Plantes: l'ultime avatar d'une scène capitale ", dans S. Houppermans (dir.), op. cit., p. 87-97.

Metka Zupancic, "Questions esthétiques et éthiques autour de la notion du mythe ", dans S. Houppermans (dir.), op. cit., p. 99-107.

\section{2}

Maria Minich Brewer, «(In)Commensurabilities: The Childhood of Events and the Shock of Encounter in Claude Simon ", dans Jean H. Duffy et Alastair B. Duncan (dir.), Claude Simon. A retrospective, Liverpool, Liverpool university press, 2002, p. 39-60.

Celia Britton, "Instant Replays : the Reintegration of Traumatic Experience in Le Jardin des Plantes", dans J. Duffy et A. B. Duncan (dir.), op. cit., p. 61-76.

Jean H. Duffy, "Truth, Verbiage and Ecriture in Le Jardin des Plantes », dans J. H. Duffy et A. B. Duncan (dir.), op. cit., p. 183-204.

Alastair B. Duncan, Claude Simon. Adventures in Words, Manchester, Manchester University Press, 1994, 204 p. Nouvelle édition revue et augmentée en 2002, chap. 8 "Le Jardin des Plantes et Le Tramway: coherence without closure".

David G. Ellison, "À partir du Jardin des Plantes: Claude Simon's Recapitulations ", dans J. H. Duffy et A. B. Duncan (dir.), op. cit., p. 135-151.

Brigitte Ferrato-Combe, "Critique et fiction dans Le Jardin de Plantes de Claude Simon ou de l'intérêt d'écrire "la marquise sortit à cinq heures" ", Recherches et travaux, $\mathrm{n}^{\circ}$ 60, 2002, p. 143-160.

Jean-Yves Laurichesse, "Passeur en eaux troubles: l'épisode du passage des armes dans Le Sacre du Printemps et Le Jardin des Plantes de Claude Simon ", dans P. Carmignani (dir.), Figures du Passeur, Perpignan, Presses universitaires de Perpignan, 2002, p. 133-158. 
Jean-Yves Laurichesse, «Écriture des ruines et métapoétique du fragment dans Le Jardin des Plantes de Claude Simon ", dans R. Ripoll (dir.), L'Écriture fragmentaire: théories et pratiques: actes du Ir Congrès international du Groupe de Recherches sur les Écritures Subversives (GRES), Perpignan, Presses universitaires de Perpignan, 2002, p. 289-301.

Mary Orr, "The Garden of Forking Paths: Intertextuality and Le Jardin des Plantes", dans J. Duffy et A. B. Duncan (dir.), op. cit., p. 118-134.

Claire de Ribaupierre, Le Roman généalogique. Claude Simon et Georges Perec, Bruxelles, La Part de l'œil, 2002.

Claudia von Wileken, "Le Jardin des Plantes de Claude Simon ou les chemins de la botanique ", dans Rabâa Abdelkefi (dir.), Travaux du groupe de recherche sur "Les écritures du moi », 1999-2001, Montpellier, université Paul Valéry Montpellier 3, 2002, p. 229-247.

\section{4}

Mireille Calle-Gruber, Le Grand Temps: essai sur l'ouvre de Claude Simon, Villeneuve d'Ascq, Presses universitaires du Septentrion (rééd. Villeneuve-d'Ascq, Presses universitaires du Septentrion, 2011).

Yves Peyré, « Claude Simon : une tension rentrée vers le visible » (entretien avec Stéphane Bikialo), dans S. Bikialo et C. Rannoux (dir.), La Licorne, n 71 (" Les Images chez Claude Simon: des mots pour le voir »), PUR, 2004, p. 93-94.

\section{5}

Maria Minich Brewer, "Pour un devenir-archives dans l'œuvre simonienne", Revue des Lettres modernes, série "Claude Simon ", n 4 ("Le (Dé)goût de l'archive "), Minard, 2005, p 31-56.

Véronique Gaultier, "Iconographie de la personne défunte: Sculpture, peinture et photographie dans les romans de Claude Simon ", Revue des Lettres modernes, série "Claude Simon ", n 4: ("Le (Dé)goût de l'archive »), Minard, 2005, p. 101-121.

Jacques Isolery "Le jardin des traces dans les romans de Claude Simon", Revue des Lettres modernes, série "Claude Simon ", n 4: ("Le (Dé)goût de l'archive "), Minard, 2005, p. 57-99.

Jean-Yves Laurichesse, "Quelque chose à dire": Éthique et poétique chez Claude Simon ", Cahiers de narratologie, n' 12, jan. 2005 (disponible en ligne à l'adresse [https://narratologie.revues.org/25]).

Jean-Yves Laurichesse, "L'illisible" à grands pas. Faire son chemin dans le texte de Claude Simon ", dans R. Ripoll (dir.), Stratégies de l'illisible, Perpignan, Presses universitaires de Perpignan, 2005, p. 43-59.

Nathalie Piégay-Gros, "Mélancolie du montage. Le Jardin des Plantes de Claude Simon », dans E. Bouju (dir.), L'Engagement littéraire, PUR, coll. "Interférence», 2005, p. 283-291. 
Ralph Sarkonak, "L'effet d'archive ", Revue des Lettres modernes, série " Claude Simon ", n 4 («Le (Dé)goût de l'archive »), Minard, 2005, p. 5-27.

\section{6}

Jean-Marie Barnaud "L'écriture et la survie. Sur une mélancolie ", Cahiers Claude Simon, $\mathrm{n}^{\circ} 2,2006$, p. 17-30, [http://associationclaudesimon.org/lecteurs/ hommages-et-temoignages/article/].

Matei Chihaya, "La haie et l'amputation. La mise en ouvre des métaphores dans Le Jardin des Plantes", dans I. Albers et W. Nitsch (dir.), Transports. Les métaphores de Claude Simon, Frankfurt am Main, Peter Lang, 2006, p. 81-98.

Jean H. Duffy, " Notice du Jardin des Plantes ", dans CE I, p. 1466-1489.

Anne-Yvonne Julien, "Histoires de feuilles: aux abords du Jardin des Plantes de Claude Simon ", dans Vincent Laisney (dir.), Le Miroir et le Chemin: L'Univers romanesque de Pierre-Louis Rey, Presses de la Sorbonne nouvelle, 2006, p. 329-338.

Jean-Yves Laurichesse, "Cendre": un avant-texte lointain du Jardin des Plantes", Cahiers Claude Simon, n 2, 2006, p. 139-146.

Dominique Viart, « Maintenant: Claude Simon », Cahiers Claude Simon, n² 2, 2006, p. 9-14, [http://associationclaudesimon.org/lecteurs/hommages-et-temoignages/article/].

\section{7}

Mokhtar Belarbi, "Auto-alterbiographie dans Les Géorgiques et Le Jardin des Plantes de Claude Simon ", dans Texte (Toronto), 41-42, 2007, p. 151-166.

Emmanuel Bouju, «Boucle épigraphique et téléologie romanesque chez Claude Simon, W.G. Sebald et Graham Swift », Fabula, 2007, [http://www.fabula.org/colloques/ document722.php].

Sophie Charlin, "Que reste-t-il du montage textuel? Efficacité du montage et résidu d'image dans Le Jardin des Plantes de Claude Simon ", Littérature, n 147, sept. 2007 , p. 38-53, [https://www.cairn.info/revue-litterature-2007-3-page-38.htm].

Anne-Yvonne Julien, "Entre Paris et l'ailleurs. Dans Le Jardin des Plantes de Claude Simon ", dans Gabrielle Chamarat et Claude Leroy (dir.), RITM, n 37 (" Le Voyage à Paris »), 2007, p. 257-270.

Antje Tennstedt, Annäherungen an die Vergangenheit bei Claude Simon und W.G. Sebald. Am Beispiel von Le Jardin des Plantes, Die Ausgewanderten und Austerlitz, Freiburg, Rombach, 2007. 


\section{8}

Claude Simon, "Plan de montage du Jardin des Plantes", dans M. Calle-Gruber (dir.), Les Triptyques de Claude Simon ou l'art du montage, Presses Sorbonne nouvelle, 2008, p. 124-126.

Joëlle Gleize, " De quelques textes hétérogènes, Leiris, Simon », dans S. Coëllier (dir.), Le Montage dans les arts auX XX et XXI siècles, Aix-Marseille, Publications de l'université de Provence, 2008, p. 117-130.

2009

Mireille Calle-Gruber, «La peur dans le fond. Une lecture du Jardin des Plantes de Claude Simon ", dans I. Matsumoto et M. Tadoroko (dir.), La Peur dans le monde moderne: aspects et perspectives, Nagoya, Éditions de l'école doctorale des langues et cultures de l'université de Nagoya, 2009, p. 8-18.

Annie Clément-Perrier, "La photographie chez Claude Simon: un objet mélancolique? ", Cahiers Claude Simon, n 5, 2009, p. 67-83, [http://associationclaudesimon.org/ressources-critiques/articles/article/clement-perrier-annie-la].

Katerine Gosselin, "Jeux de fragmentation dans Le Jardin des Plantes et Le Tramway de Claude Simon », @nalyses, n 4(2) ("Fiction et réel»), 25 mars 2009, [https:// uottawa.scholarsportal.info/ojs/index.php/revue-analyses/article/view/635.

Patrick Suter, " "Reportage" et "écriture" chez Claude Simon (Les Géorgiques et Le Jardin des Plantes) ", Etudes Littéraires (Québec), n 40/3, automne 2009, p. 141-155.

2010

Bérénice Bonhomme, Claude Simon: la passion-cinéma, Villeneuve d'Ascq, Presses universitaires du Septentrion, coll. "Claude Simon », 2010, en particulier p. 384-409.

Isabelle Serça, "Du temps perdu au temps suspendu ", dans Philippe Chardin (dir.), Originalités proustiennes, Kimé, coll. " Détours littéraires », 2010, p. 263-281.

Cécile Yapaudjan-Labat, "Voir Berlin et écrire. Images de ruines dans Le Jardin des Plantes de Claude Simon et La Reprise d'Alain Robbe-Grillet ", dans Roger-Michel Allemand et Christian Milat (dir.), Alain Robbe-Grillet: balises pour le XXI siècle. Ottawa/Paris, Presses universitaires d'Ottawa/Presses Sorbonne nouvelle, 2010, p. 424-434.

Cécile Yapaudjan-Labat, Écriture, deuil et mélancolie-Les derniers textes de Samuel Beckett, Robert Pinget et Claude Simon, Classiques Garnier, coll. "Études de littérature des $\mathrm{Xx}^{\mathrm{e}}$ et $\mathrm{XXI}^{\mathrm{e}}$ siècles ", 2010. 


\section{1}

Laurence Cadet, De Claude Simon à Proust: le miroitement des textes, Champion, 2011.

Paul Dirkx et Pascal Mougin, "Introduction ", dans P. Dirkx et P. Mougin (dir.), Claude Simon Situations, Lyon, ENS Éditions, 2011, p. 11-23.

Chiara Falangola, "Écrire (d')après Proust: citations et influences proustiennes dans la deuxième partie du Jardin des Plantes de Claude Simon ", @nalyses, sept. 2011, [https://uottawa.scholarsportal.info/ojs/index.php/revue-analyses/article/view/645].

Yona Hanhart-Marmor, "Texte et contexte: perspectives esthétiques. L'exemple du Jardin des Plantes ", dans P. Dirkx et P. Mougin (dir.), op. cit., p. 163-178.

Sabrina Parent, Poétiques de l'événement. Claude Simon, Jean Rouaud, Eugène Savitzkaya, Jean Folain, Jacques Réda, Classiques Garnier, coll. "Études de littérature des $\mathrm{xx}^{\mathrm{e}}$ et $\mathrm{XXI}^{\mathrm{e}}$ siècles ", 2011 (en particulier la section "Le Jardin des Plantes de Claude Simon: de l'imminence à l'immanence ", p. 181-198).

Michèle Touret, "L'autre moitié de l'orange, la guerre cinquante ans après ", dans P. Dirkx et P. Mougin (dir.), op. cit., p. 143-159.

Cécile Yapaudjan-Labat, "Fantômes en coulisses dans Le Jardin des Plantes: Claude Simon à la poursuite de Dostoïevski ", dans Anne Chamayou et Nathalie Solomon (dir.), Fantômes d'écrivains, Perpignan, Presses universitaires de Perpignan, 2011, p. 177-196.

\section{2}

Llewellyn Brown « Voile et structuration phallique dans Le Jardin des Plantes de Claude Simon ", Revue des Lettres modernes, série "Claude Simon ", n 6 (" La Réception critique »), Caen, Minard, 2012, p. 239-257.

Paul Dirkx, "Le corps autographe: nouvelles hypothèses sur Le Jardin des Plantes de Claude Simon ", dans Björn-Olav Dozo, Anthony Glinoer et Michel Lacroix (dir.), Imaginaires de la vie littéraire, Rennes, PUR, 2012, p. 301-312.

Jean-Yves Laurichesse, "La critique simonienne et le référent. L'insistance du réel ", Revue des Lettres modernes, série "Claude Simon ", n 6 ("La Réception critique »), 2012, p 97-133.

Emilie Lucas-Leclin, "Image et discontinuité narrative dans Le Jardin des Plantes de Claude Simon et La Perte de l'image de Peter Handke ", dans Alison Boulanger, Chiara Nannicini et Alice Pintiaux, Ruptures du récit. Essais sur la discontinuité narrative, Le Manuscrit, 2012, p. 79-99.

\section{3}

Wolfram Nitsch, "De l'avion à l'abîme: vues aériennes chez Claude Simon », dans J.-Y. Laurichesse (dir.), Claude Simon géographe, Classiques Garnier, 2013, 
p. 185-199, [http://www.canalu.tv/producteurs/universite_toulouse_ii_le_mirail/ dossier_programmes/colloques/claude_simon_geographe/].

Bertrand Westphal, "Promenade géocritique à travers Le Jardin des plantes de Claude Simon ", dans J.-Y. Laurichesse (dir.), op. cit., p. 31-41.

\section{4}

Isabelle Diu et Sophie Lisiewicz, «Le fonds Claude Simon à la Bibliothèque littéraire Jacques Doucet ", dans Melina Balcazar Moreno, Mireille Calle-Gruber, SarahAnaïs Crevier Goulet et Anaïs Frantz (dir.), Claude Simon, Les Vies de l'archive, Dijon, Éditions universitaires de Dijon, 2014, p 159-168.

Marie Hartmann, «Éclats du passé. Claude Simon, Le Jardin des Plantes », dans Brigitte Diaz, Franziska Meier et Francine Wild (dir.), Les Héritages littéraires dans la littérature française (XVI -XXe siècle), Classiques Garnier, «Rencontres », 2014, p. 251-265.

Kaya Mümtaz, "Le Jardin des Plantes et Mon Nom est Rouge: hypotypose, ekphrasis et thèmes communs", Nouvelles études francophones, n² 29(1), 2014, p. 27-41.

\section{5}

Paul Dirkx, «Une politique autonome: l'érotisme littéraire. Claude Simon, Le Jardin des Plantes (1997) ", dans J. Dubois (dir.), Sexe et pouvoir dans la prose française contemporaine, Liège, Presses universitaires de Liège, 2015, p. 173-187.

Katerine Gosselin, "À contretemps du Nouveau roman. Réécriture et relecture dans Le Jardin des Plantes de Claude Simon ", Tangence, $\mathrm{n}^{\circ}$ 107, 2015, p. 33-54, [https:// www.erudit.org/fr/revues/tce/2015-n107-tce02164/1033949ar/].

Katerine Gosselin, "Comme si je lui parlais dans une langue inconnue", le rapport entre ouvre et discours chez Claude Simon », Europe, n 1033 ("Claude Simon »), mai 2015, p 92-104.

Patrick Longuet, «La meilleure part », Europe, n 1033 ("Claude Simon »), mai 2015, p. 12-20.

Hans Peter Lund, "Traduire Claude Simon en danois: Le Jardin des Plantes et Le Tramway", Cahiers Claude Simon, n 10, 2015, p. 129-140.

Marie-Albane Watine, «La transcription d'un texte critique dans le roman: ambiguïté et désengagement théorique dans Le Jardin des Plantes de Claude Simon ", dans Marianne Bouchardon et Myriam Dufour-Maître, L'Ombre dans l'euvre: la critique dans l'ouvre littéraire, Classiques Garnier, 2015, p. 209-224.

Ilias Yocaris et David Zemmour, "Un texte convenablement composé”, Du local au global dans les romans de Claude Simon », Europe, n 1033 ("Claude Simon »), mai 2015, p. 198-210. 


\section{6}

Alina Cherry, Claude Simon: Fashioning the Past by Writing the Present, Madison/ Teaneck, Fairleigh Dickinson University Press, 2016.

Christine Genin, "Lire Claude Simon lisant Proust», Tangence, n 112, 2016, p. 109-131.

Joëlle Gleize, "Dynamiques du montage: vers une adhésion sensible", Tangence, $\mathrm{n}^{\circ} 112$, p. 47-62.

Olivier Kahn, "Singularité de la génétique simonienne dans Le Jardin des Plantes", dans Martine Créac’h et Aurélie Renaud (dir.), Claude Simon \&..., actes du séminaire " Jeunes chercheurs » de l'Association des Lecteurs de Claude Simon du 3 juin 2016, [https://www.fabula.org/colloques/document3502.php].

Hannes de Vriese, "L’Acacia, 25 ans de critique ", Cahiers Claude Simon, n 11, 2016, p. $27-52$.

\section{7}

Paul Dirkx, "Claude Simon: corps de l'écrivain et érotisme littéraire. Le Jardin des Plantes et Triptyque ", Cahiers Claude Simon, n 12, 2017, p 145-160. 